\title{
Crossing technological frontiers in radioguided intervention
}

\author{
Renato A. Valdés Olmos ${ }^{1,2,3} \cdot$ Sergi Vidal-Sicart ${ }^{4} \cdot$ Fijs WB van Leeuwen ${ }^{1}$
}

Published online: 9 August 2016

(C) Springer-Verlag Berlin Heidelberg 2016

Following its incorporation into the management of cutaneous melanoma and breast cancer, the sentinel node (SN) procedure has been the corner stone of radioguided surgery for the last 25 years [1]. Based upon the use of radioactive colloidal tracers administered at the tumour site and their prolonged $\mathrm{SN}$ retention, gamma probes were incorporated into the SN biopsy in the early years of the procedure [2]. These devices were not only able to count radioactivity in the surgical field, but also to provide audible signals proportional to the counting rate in the SNs. In fact, gamma probe counting constituted the acoustic component of the SN identification procedure in the operating room and was introduced to complement the process of visual detection of superficial lymphatic ducts and SNs using blue dyes, as implemented by surgeons. In the evolution of the procedure, this acoustic/visual approach meant a step forward in comparison with the original purely optical approach of Morton et al. [3].

The possibility of combining visual and acoustic signals during the intraoperative SN procedure constituted a paradigm

This Editorial Commentary refers to the article http://dx.doi.org/10.1007 /s00259-016-3443-0

Renato A. Valdés Olmos

R.A.Valdes_Olmos@lumc.nl

1 Interventional Molecular Imaging Laboratory, Department of Radiology, Leiden University Medical Centre, P.O. Box 9600, 2300 RC Leiden, The Netherlands

2 Nuclear Medicine Section, Department of Radiology, Leiden University Medical Centre, Leiden, The Netherlands

3 Nuclear Medicine Department, Netherlands Cancer Institute - Antoni van Leeuwenhoek Hospital, Amsterdam, The Netherlands

4 Nuclear Medicine Department, University Hospital Clínic Barcelona, Barcelona, Spain shift in the technological sense and provided the fundament for new portable imaging modalities [4]. This evolution is illustrated by the development of portable technologies including small gamma cameras for high resolution planar imaging particularly useful to localize SNs in the vicinity of the injection site [5] and gamma probe- or handheld camera-based devices for three-dimensional (3D) intraoperative imaging [6]. These tools have reinforced the visual component in the guidance of surgical excision in areas of complex anatomy [7]. A next step has been the combination of these nuclear medicine devices with allied technologies, such as near-infrared (NIR) cameras when fluorescent detection is added to the radioguided procedure [8], or the recent introduction of cameras for Cerenkov-luminescence imaging [9].

\section{Synergism of nuclear medicine with allied technologies}

In recent years, some non-nuclear medicine technologies have been combined with the conventional portable gamma devices to improve intraoperative detection, principally in malignancies with complex anatomical lymphatic drainage. One example is the detection of cervical and pelvic SNs using portable gamma cameras and NIR-cameras. The introduction of fluorescent dyes to the $\mathrm{SN}$ procedure makes it possible today to use this modality in addition to gamma ray detection. Although various groups have validated intraoperative fluorescent imaging for $\mathrm{SN}$ as an independent modality, its limitation to generate adequate preoperative lymphatic mapping, principally in depth, has led to the delineation of two combined approaches both requiring the use of nuclear medicine for preoperative lymphatic mapping. The first one is based on the principle "two modalities, two signatures," and involves the use of separate detection devices and agents with the 
radioactive tracer preoperatively administered and the fluorescent dye intraoperatively injected. The second approach, incorporating fluorescence to the radioactive tracer, is based upon the concept "two modalities, one signature" using a hybrid tracer, which enables nuclear physicians to perform preoperative lymphatic mapping including single photon emission computed tomography/computed tomography (SPECT/CT), and subsequently surgeons in the operating room to combine portable gamma detection with fluorescence near-infrared (NIR)-camera imaging. On a clinical basis, the most extensively validated hybrid tracer currently is ICG- ${ }^{99 \mathrm{~m}} \mathrm{Tc}$-nanocolloid, which showed $100 \%$ reproducibility when compared with the parental ${ }^{99 \mathrm{~m}} \mathrm{Tc}$-nanocolloid [10], and subsequently has been introduced for SN biopsy in melanoma [11], breast cancer [12], oral malignancies [13], vulvar cancer [14] and prostate cancer [15]. This hybrid approach combining radioguidance technology with fluorescence, whereby the strengths of the imaging technology remain preserved and are complemented by the advantages of the other modality, provides perspective for other complex radioguided procedures in the future, extending the role of nuclear medicine.

\section{Fusion of freehand SPECT and ultrasound}

The combined use of gamma devices and NIR-cameras in a certain sense formed the vanguard of new approaches combining nuclear medicine tools with allied technologies to improve image-guided intervention. In this issue of Eur J Nucl Med Molecular Imaging, Bluemel C et al. report the fusion of freehand SPECT (fhSPECT) and ultrasound (US) for preoperative SN localization. Their signal integration made it possible to differentiate radioactive lymph nodes considered as SNs from other lymph nodes not directly draining from the tumour site. To accomplish this, both handheld devices were tracked using retro-reflecting fiducial markers that facilitated topographical positioning of their findings in relation to a similar fiducial placed on the patient. The primary aim of the study was to evaluate infrared stereo tracked fusion of freehand SPECT performed with a handheld gamma camera and ultrasound for anatomical co-registration of SNs in a group of patients including 118 breast cancers, 30 cutaneous melanomas and three vulvar cancers [16]. Fused fhSPECT/US findings were directly compared to preoperative lymphatic mapping including lymphoscintigraphy and SPECT/CT. Although measurements in obese patients and the existence of multiple lymph nodes led to a limited overall co-registration (72\%), tracked fhSPECT/US is also a new illustration of a hybrid approach to explore alternative modalities for SN identification. The final objective of this technological approach needs to be looked for in the potential reduction, or eventually in the replacement, of the conventional surgical SN procedure by this novel method. This was the original approach in the pilot publications of Okur A et al. [17] and de Bree R et al. [18] in which fine-needle aspiration cytology (FNAC) was used in combination with fused fhSPECT/US. However, it is known that the sensitivity of FNAC sampling is limited and to match the effectiveness of surgical SN biopsy, most probably core needles and larger $\mathrm{SN}$ aspiration samples will be required. As mentioned by Bluemel $\mathrm{C}$ et al., core needle biopsy guided by tracked fhSPECT/US has recently been applied in 38 patients in a pilot German multicentre study and appears to be feasible [19].

\section{Future multidisciplinary clinical and technological challenges}

In fact, the combination of both handheld devices as validated by Bluemel $\mathrm{C}$ et al. matches a functional signal (nuclear medicine) to a morphological one (ultrasound). This tracked fusion potentially enables nuclear physicians to facilitate targeted SN biopsy outside of the operation room. This hybrid approach follows not only the combined use of gamma-ray devices and NIR-cameras in the operating room, but also the trend to integrate functional and morphological modalities in large devices like SPECT/CT and positron emission tomography (PET)/CT for diagnostic and interventional imaging.

Together with the intraoperative modernization of the SN procedure, preoperative imaging also evolved from a single nuclear medicine modality like lymphoscintigraphy to a hybrid approach using SPECT/CT. Thanks to the improvement of its CT component, this latter hybrid technology has provided an anatomical environment with specific landmarks in the fused SPECT/CT images for localizing SNs. This improved imaging procedure transformed the $\mathrm{SN}$ visualization paradigm, incorporating a 3D roadmap useful not only to search for, but also to recognize SNs during open surgery or laparoscopy. A similar evolution was observed for total body scintigraphy when SPECT/CT and PET/CT became feasible following systemic administration of tumour-seeking radiotracers. At present, in cases suspected of cancer recurrence due to the elevation of tumour markers during follow-up, SPECT/ $\mathrm{CT}$ and principally PET/CT can detect lesions considered as potential targets and simultaneously can provide real 3D anatomical roadmaps to guide resection or intervention for diagnostic or therapeutic purposes [20]. The integration of CT with SPECT and PET resulted in a necessary adjustment in the training schedules of nuclear physicians and a close collaboration with radiologists.

With the incorporation of other allied technologies (e.g. fluorescence detection, ultrasound) to the original radioguided techniques, the multidisciplinary concept has been extended to these fields. When the SN procedure was introduced in cancer care, a multidisciplinary clinical approach involving nuclear physicians, surgeons and pathologists was mandatory 
to establish good practice skills. The renewed technological diversification of recent years asks for new professional requirements for the disciplines involved in radioguided interventions, at least in centres with a high volume of these procedures and advanced clinical indications. A necessary knowledge concerning allied technologies and the possibility of training for residents and specialists in centres of expertise needs to become matter of discussion in future educational programmes.

The crossing of technological frontiers in radioguided intervention defines new challenges for the coming years. When adaptive to the changing environment, as demonstrated in the study of Bluemel $\mathrm{C}$ et al., nuclear medicine may continue to play a leading role among the clinical disciplines involved in $\mathrm{SN}$ procedures and other image-guided interventions.

\section{Compliance with ethical standards}

Conflicts of interest All authors declare that they have no conflict of interest.

Ethical approval This article does not contain any studies with human participants or animal performed by any of the authors.

\section{References}

1. Valdés Olmos RA, Vidal-Sicart S. Introducing new perspectives in radioguided intervention. Clin Transl Imaging. 2016. doi:10.1007 /s40336-016-0177-8.

2. Krag DN, Weaver DL, Alex JC, Fairbank JT. Surgical resection and radiolocalization of the sentinel lymph node in breast cancer using a gamma probe. Surg Oncol. 1993;2:335-9.

3. Morton DL, Wen DR, Wong JH, Economou JS, Cagle LA, Storm FK, et al. Technical details of intraoperative lymphatic mapping for early stage melanoma. Arch Surg. 1992;127:392-9.

4. Valdés Olmos RA, Vidal-Sicart S, Nieweg OE. SPECT-CT and real-time intraoperative imaging: new tools for sentinel node localization and radioguided surgery. Eur J Nucl Med Mol Imaging. 2009;36:1-5.

5. Tsuchimochi M, Hayama K. Intraoperative gamma cameras for radioguided surgery: technical characteristics, performance parameters, and clinical applications. Phys Med. 2013;29:126-38.

6. Valdés Olmos RA, Vidal-Sicart S, Nieweg OE. Technological innovation in the sentinel node procedure: towards 3-D intraoperative imaging. Eur J Nucl Med Mol Imaging. 2010;37:1449-51.

7. Valdés Olmos RA, Vidal-Sicart S, Giammarile F, Zaknun JJ, van Leeuwen FWB, Mariani G. The GOSTT concept and hybrid mixed/ virtual/augmented reality environment radioguided surgery. Q J Nucl Med Mol Imaging. 2014;58:207-15.
8. van Leeuwen FW, Valdés Olmos R, Buckle T, Vidal-Sicart S. Hybrid surgical guidance based on the integration of radionuclear and optical technologies. Br J Radiol. 2016;89(1062):20150797.

9. Thorek DL, Riedl CC, Grimm J. Clinical Cerenkov luminescence imaging of (18)F-FDG. J Nucl Med. 2014;55:95-8.

10. Brouwer OR, Vermeeren L, Klop WMC, Balm AJM, van der Poel HG, van Rhijn BW, et al. Comparing the hybrid fluorescentradioactive tracer indocyanine green-99mTc-nanocolloid with 99mTc-nanocolloid for sentinel node identification: a validation study using lymphoscintigraphy and SPECT/CT. J Nucl Med. 2012;53:1034-40.

11. van den Berg NS, Brouwer OR, Schaafsma BE, Mathéron HM, Klop WM, Balm AJ, et al. Multimodal surgical guidance during sentinel node biopsy for melanoma: combined gamma tracing and fluorescence imaging of the sentinel node through use of the hybrid tracer indocyanine green- $(99 \mathrm{~m}) \mathrm{Tc}$-nanocolloid. Radiology. 2015;275:521-9.

12. Schaafsma BE, Verbeek FP, Rietbergen DD, van der Hiel B, van der Vorst JR, Liefers GJ, et al. Clinical trial of combined radio- and fluorescence-guided sentinel lymph node biopsy in breast cancer. Br J Surg. 2013;100:1037-44.

13. Van den Berg NS, Brouwer OR, Klop WMC, Karakulluckcu B, Zuur CL, Tan IB, et al. Concomitant radio- and fluorescenceguided sentinel lymph node biopsy in squamous cell carcinoma of the oral cavity using ICG-99mTc-nanocolloid. Eur J Nucl Med Mol Imaging. 2012;39:1128-36.

14. Mathéron HM, van den Berg NS, Brouwer OR, Kleinjan GH, van Driel WJ, Trum JW, et al. Multimodal surgical guidance towards the sentinel node in vulvar cancer. Gynecol Oncol. 2013;131:720-5.

15. Kleinjan GH, van den Berg NS, Brouwer OR, de Jong J, Acar C, Wit EM, et al. Optimisation of fluorescence guidance during robotassisted laparoscopic sentinel node biopsy for prostate cancer. Eur Urol. 2014;66:991-8.

16. Bluemel C, Safak G, Cramer A, Wöckel A, Gesierich A, Hartmann E, et al. Fusion of freehand SPECT and ultrasound: First experience in preoperative localization of sentinel lymph nodes. Eur J Nucl Med Mol Imaging. 2016.

17. Okur A, Hennersperger C, Runyan B, Gardiazaball J, Keicher M, Paepke S, et al. FhSPECT-US guided needle biopsy of sentinel lymph nodes in the axilla: is it feasible? Med Image Comput Comput Assist Interv. 2014;17(Pt 1):577-84.

18. de Bree R, Pouw B, Heuveling DA, Castelijns JA. Fusion of freehand SPECT and ultrasound to perform ultrasound-guided fineneedle aspiration cytology of sentinel nodes in head and neck cancer. Am J Neuroradiol. 2015;36:2153-8.

19. Paepke S, Pfob C, Ohlinger R, Gruber I, Thill M, Blohmer J, et al. Abstract P3-01-16: can sentinel node staging be performed using a minimally invasive needle-biopsy? Results of German multicentric pilot study. Proceedings of the Thirty-Eighth Annual CTRC-AACR San Antonio Breast Cancer Symposium: 2015 Dec 8-12; San Antonio, TX Philadelphia (PA): AACR; Cancer Res 2016;76: Abstract nr P3-01-16; 2016. p. P3-01-16.

20. Solomon SB, Cornelis F. Interventional molecular imaging. J Nucl Med. 2016;57:493-6. 